NOTICE: This is the author's version of a work that was accepted for publication in Forensic Science International. Changes resulting from the publishing process, such as peer review, editing, corrections, structural formatting, and other quality control mechanisms may not be reflected in this document. Changes may have been made to this work since it was submitted for publication. A definitive version was subsequently published in Forensic Science International, Vol. 228, Issues 1-3. (2013). doi: 10.1016/j.forsciint.2013.01.032 


\section{Characterisation of Chemical Component Migration in Automotive Paint by Synchrotron Infrared Imaging}

Mark Maric ${ }^{1}$, Wilhelm van Bronswijk ${ }^{1}$, Simon W. Lewis ${ }^{1 *}$, Kari Pitts ${ }^{2}$, Danielle E. Martin ${ }^{3}$

${ }^{1}$ Department of Chemistry, Curtin University of Technology, GPO Box U1987, Perth, Western Australia 6845, Australia;

${ }^{2}$ Chem Centre, Bentley Delivery Centre, PO Box 1250, Western Australia 6983, Australia;

${ }^{3}$ Australian Synchrotron, 800 Blackburn Road, Clayton, Victoria 3168, Australia.

*Author for correspondence:

Simon W. Lewis

Department of Chemistry, Curtin University

GPO Box U1987 Perth, Western Australia 6845

E-mail: S.Lewis@curtin.edu.au Tel: +61 (08) 92662484 


\begin{abstract}
Synchrotron infrared chemical imaging was employed to examine and assess the extent of interlayer component migration within multilayer automotive paint samples, with a particular emphasis on the cross-linking additive melamine. Two dimensional infrared chemical images revealed that melamine consistently diffuses in select paint samples from the underlying basecoat into the outermost clear coat layer. Pigments from the basecoat were also found to migrate into the adjoining layers. This is significant as the relative abundance of both melamine and pigments will vary greatly depending upon the region of the layer analysed. This component migration will undoubtedly impact the information gleaned from a questioned sample via library searching software or multivariate statistics. As a result, appropriate analytical protocols will need to be utilised to mitigate the effects of interlayer pigment and melamine diffusion, so as to afford a true representation of the composition of the coating for forensic identification purposes.
\end{abstract}

Keywords: trace evidence; automotive paint; infrared imaging; synchrotron; melamine

\title{
Introduction
}

Automotive paint, in the form of chips or smears, is one of the most commonly encountered forms of trace evidence in automobile collisions, hit and run accidents or other vehicle related incidents. ${ }^{[1,2]}$ Automotive paint is a complex multicomponent system designed to protect the frame of the vehicle and achieve the desired colour and finish. ${ }^{[3]}$ Most commonly, the first layer applied to the body of the vehicle is the electrocoat primer which provides corrosion resistance. A primer surfacer is then applied to conceal surface imperfections, so as to form a smooth foundation amenable to the application of the basecoat. Following the application of the primer surfacer, a pigment-containing layer known as the basecoat is applied to achieve the desired colour and effect. This layer also contains the uncoated or coated aluminium 
flakes, which gives cars their metallic appearance. The final coat applied in the automotive finishing process is the clear coat, which is an unpigmented layer designed to protect the underlying basecoat of the vehicle from ultraviolet degradation and weathering. ${ }^{[1-4]}$ Each layer has a characteristic function and is comprised of a distinctive permutation of binders, resins, pigments and additives. ${ }^{[3]}$

The analytical approach towards forensic paint examination relies on the analysis of each layer via a combination of microscopic and spectroscopic techniques. ${ }^{[5]}$ Infrared (IR) spectroscopy is unequivocally the most powerful, non-destructive technique available for the characterisation of paint, providing information pertaining to the composition and relative abundance of binder, resin and pigment components. ${ }^{[6]}$ The chemical information obtained from the spectroscopic characterisation of paint evidence may be employed to either; perform questioned vs. known comparisons, or to procure investigative leads by identifying the source of the vehicle either by a library search of a database or by the use of multivariate statistics such as principal component analysis (PCA) and linear discriminant analysis (LDA). Chemometrics is a form of pattern recognition, enabling patterns and relationships to be discerned in the data that would otherwise remain elusive. Ultimately, this form of statistical analysis may allow the user to obtain information about the vehicle from an unknown paint sample (such as make, model or provenance), based upon its classification into one of the prespecified groupings.

Fourier transform infrared (FTIR) microspectroscopy is heavily relied upon in the analysis of multicomponent systems such as automotive paint, as it allows the user to sequentially examine all layers of a cross-sectioned coating without the need for individual separation of layers or complex sample preparation. ${ }^{[7,8]}$ The characterisation of automotive paint specimens using FTIR spectroscopy is not a novel concept, with several sampling techniques including transmission, reflection, transflection, diffuse reflectance and attenuated total 
reflection (ATR) being reported in the open literature. ${ }^{[7,9-12]}$ ATR FTIR spectroscopy is a technique which minimises the need for sample preparation since only contact between the specimen surface and ATR crystal is required, allowing rapid characterisation of the surface of the outermost layer (i.e. clear coat). ${ }^{[13]}$ Recently, Edmondstone and co-workers utilised ATR FTIR spectroscopy to characterise and visually discriminate between a substantial collection of automotive clear coats. ${ }^{[14]}$ Similarly, a more recent study conducted by the authors Maric et al, employed ATR FTIR spectroscopy in conjunction with multivariate statistics to assess the inherent diversity of a large population of clear coats. PCA performed on the spectral data revealed distinct classes which can be attributed to the source of the vehicle and in some instances the manufacturer. ${ }^{[15]}$ LDA gave excellent discrimination between the classes with all of the samples being correctly classified. ${ }^{[15]}$ Consequently, the statistical model may be of significant assistance in procuring investigative leads and presenting evidence in court. However, in order to further improve the generated model, information concerning the homogeneity of the layers and interlayer migration of components needs to be assessed, to develop more suitable analytical protocols for the characterisation of paint evidence. As such, IR chemical imaging has been employed to afford 2-dimensional spatial and spectral information of multi-layered automotive paint cross-sections.

IR chemical imaging has enormous potential in the field of forensic science, owing to the fact that chemical and spatial information can be obtained about a sample synchronously. Previous research by Flynn et al has demonstrated that focal plane array (FPA) detectors can be employed in the hyperspectral imaging of automotive paint chips. ${ }^{[16]}$ The main advantage of FPA IR chemical imaging in the analysis of automotive paint chips is that the simultaneous acquisition of spectra can be accomplished from all the layers concurrently. This makes the method extremely time effective when compared to conventional IR 
microspectroscopy using single element IR detectors. ${ }^{[17]}$ However, FPA FTIR spectroscopy using a conventional globar source is still hampered by limited spatial resolution, which is detrimental in many instances and particularly where samples contain relatively low abundances of constituents in close proximity. ${ }^{[17]}$

Use of synchrotron IR microspectroscopy is able to overcome these shortcomings. Although maps are again time limited by use of single element IR detectors, synchrotron IR radiation is highly collimated and in the order of 1000 times brighter than conventional globar IR sources. This enables spectra to be acquired at significantly improved signal-to-noise ratios and at diffraction-limited spatial resolutions, which is of particular significance when dealing with complex samples such as automotive paint evidence as it allows for the differentiation and distribution analysis of key vibrational bands corresponding to binders, resins, pigments and additives in the different layers.

The aim of this study was to employ synchrotron FTIR microspectroscopy to assess the extent of component migration amidst the layers of automotive paint samples, with particular emphasis on the cross-linking additive melamine. Melamine is a reactive chemical component frequently utilised by automotive manufacturers in their formulation to initiate cross-linking of the polymeric resins during the curing process, to form extended networks, thus affording a hard and durable coating. ${ }^{[3]}$ Whilst a number of analogous amino crosslinkers are occasionally employed, melamine is undeniably the most commonly encountered cross-linking additive in automotive paint systems. ${ }^{[3]}$ Previous research has revealed that low molecular weight UV absorbers and hindered amine light stabilisers are capable of migrating between the clear coat and basecoat layers during paint curing. ${ }^{[18,19]}$ It is therefore also feasible that low molecular weight cross-linkers (i.e. melamine) and polymeric additives may also readily migrate between the layers. It is recognised by the forensic community that 
interlayer migration of melamine can occur from the base coat into the clear coat, however, at this point in time there is no direct supporting evidence available in the literature.

\section{Materials and Methods}

\section{Sample Preparation:}

All automotive paint exemplars were obtained from a sunroof fitting company (Prestige Sunroofs WA) from panels removed during sunroof installation. The make, model, year and vehicle identification number (VIN) of each vehicle was recorded. A scalpel was employed to pry paint chips off the underlying metal, ensuring all layers were present in the sample. The chips of paint were then sandwiched between two rigid pieces of plastic prior to transverse sectioning with a microtome equipped with a stainless steel blade. Samples were not embedded in a resin prior to microtoming as previous research indicated the infiltration of the embedding media into the paint chips, leading to interference bands in the resultant IR spectra. ${ }^{[20]}$ The microtomed cross sections obtained from all paint samples were $8 \mu \mathrm{m}$ thick. The paint sections were then separated from the plastic and pressed flat between two microdiamond cell windows (Thermo Scientific).

Analysis:

All experiments were conducted on the IR microspectroscopy (IRM) beamline of the Australian Synchrotron, Melbourne, Australia. The mechanics of the IRM beamline are outlined in detail by Creagh et al. ${ }^{[21]}$ Briefly, the beamline consists of a Bruker Vertex V80v FTIR spectrometer equipped with a liquid nitrogen cooled narrow-band mercury cadmium telluride (MCT) detector in conjunction with a Bruker Hyperion 2000 microscope (Bruker Optik GmbH, Ettlingen, Germany). The microscope is equipped with a motorised sample stage which allows spectral mapping of regions of interest, and an atmospheric purge box to 
minimise background variation over the mapping time. The paint sections were mapped in transmission mode using an X-Y step size of $2.5 \mu \mathrm{m}$, with a $5 \mu \mathrm{m}$ x $5 \mu \mathrm{m}$ sampling aperture. Spectra were acquired over the range of $3900-730 \mathrm{~cm}^{-1}$ at a spectral resolution of $4 \mathrm{~cm}^{-1}$ with 64 co-added scans. 2-dimensional false colour chemical maps were then generated for specific IR bands of interest by correlating integrated peak areas to specific positions in the measured grid. Data acquisition and processing was performed via Bruker Opus software (version 6.5).

Spectral Processing:

All IR spectra were linearly baseline corrected and range normalised. IR transmission spectra obtained from thin polymeric films such as automotive paint cross-sections are convoluted by the presence of interference fringes, which often mask small spectral features and make quantification of components problematic. Consequently, interference fringes were removed from the pre-processed spectra following spectral subtraction of a sinusoidal wave with the same frequency as the fringes from the original data.

\section{Results and Discussion}

Figure 1 depicts the optical micrograph of an area of interest taken from a cross-sectioned paint chip obtained from a Toyota Camry. Interestingly, the image of the paint section clearly reveals bleeding of the red organic pigment from the base coat into the clear coat, as indicated by the diffusion of the red colour across the base/clear coat interface. In this particular instance, the red pigment represents a broad class of pigments known as diketopyrollo pyrroles, which was identified by the presence of two dominant characteristic IR absorption bands at $c a .1641$ and $1605 \mathrm{~cm}^{-1}$ in the resultant IR spectra of the basecoat. ${ }^{[22]}$ 
Figure 1: Optical micrograph of an automotive paint cross section obtained from a red Toyota Camry.

This migration was confirmed spectroscopically in the corresponding 2-dimensional chemical map shown in figure 2. The map displays a spatial distribution pattern for the amide stretch $\left(1641 \mathrm{~cm}^{-1}\right)$ and is comparable with the observed visible migration of the pigment. In this particular instance, it is evident that relatively strong absorption of the pigment amide stretch persists well after the base/clear coat interface, adding further credence to the notion of interlayer migration of paint components.

$<$ Figure 2>

Figure 2: 2-dimensional FTIR chemical image from a paint cross-section (Toyota Camry) following integration of the amide stretching vibrational band of the organic pigment $(\sim 1641$ $\left.\mathrm{cm}^{-1}\right)$. The pink regions of the contour map infer areas of high pigment abundance, whilst the blue zones are characteristic of regions with low or negligible pigment concentrations.

To further investigate the extent of interlayer component migration, figure 3 shows a 2dimensional chemical map demonstrating the melamine abundance across the sample, as melamine is also a significant basecoat constituent. The map was produced by integrating the band $c a .1550 \mathrm{~cm}^{-1}$, which is indicative of an in-plane triazine ring stretch for melamine. ${ }^{[6]}$ The main diagnostic peak for melamine, which occurs near $815 \mathrm{~cm}^{-1}$ and is characteristic for the out-of-plane triazine ring deformation, could not be utilised because it is too near the lower limit of the spectral cut-off for the MCT detector.

Figure 3 clearly shows a significant decrease in melamine abundance in the clear coat of the paint section from the base/clear coat boundary. In particular, the initial $20 \mu \mathrm{m}$ of the clear 
coat displays a strong IR response for melamine, which substantially diminishes when approaching the surface of the clear coat.

$<$ Figure 3 $>$

Figure 3: 2-dimensional FTIR chemical image from a paint cross-section (Toyota Camry) following integration of the in-plane triazine $\left(1550 \mathrm{~cm}^{-1}\right)$ band of melamine.

Similarly, figure 4 shows the intensity distribution of melamine across a paint section obtained from a Mazda 6. In this instance, the IR response of melamine is strong in the primer surfacer and base coat but consistently and incrementally decreases through the clear coat. This infers that the melamine additive has migrated from the base coat before subsiding halfway through the clear coat. It is worth noting the migration of melamine was only unequivocally observed in specific Japanese (i.e. Toyota, Mazda and Mitsubishi) vehicles which naturally have low melamine concentrations in the clear coat, thereby allowing subtle variations in the melamine distribution to be readily discerned. It should also be noted that this diffusion effect was only observed with melamine and low molecular weight organic pigments (i.e. diketo-pyrollo pyrolles) and was not seen with any of the other typical polymeric resins and additives, such as acrylics and styrene, present in the coating. It is proposed that the diffusion of paint components is size mediated, which corroborates the notion that large polymeric species (i.e. acrylic) are incapable of interlayer migration, whilst small low molecular weight additives and pigments are capable of readily diffusing between coatings.

The observed migration of melamine is believed to be specifically due to the manner in which the vehicle is painted. Recent progression in automotive coating technology has resulted in the development of a wet paint system, which is a one-step baking and drying 
method consisting of the successive application of the primer surfacer, base and clear coat all whilst wet. ${ }^{[23]}$ This method employs water based paints thereby significantly reducing the emission of volatile organic compounds. Moreover by eliminating the required drying process after every coat, carbon dioxide production and energy consumption greatly diminishes. ${ }^{[23]}$ This technology was initially developed by Mazda and is likely to have been instituted by other domestic manufacturers as a more environmentally friendly automobile coating process. It is believed that this wet paint system is directly responsible for the interlayer infiltration of melamine, which is further substantiated by the fact that this effect is only observed in specific Japanese vehicles where this painting process is implemented.

\section{$<$ Figure $4>$}

Figure 4: 2-dimensional FTIR chemical image of a paint cross section from a Mazda 6 contrasted using the integrated absorbance of the in-plane triazine $\left(\sim 1550 \mathrm{~cm}^{-1}\right)$ band of melamine.

The implications of these results are extremely significant. Based upon the extent of melamine dissemination observed from the underlying layers and into the clear coat, it is evident that caution must be reserved when characterising specific individual layers (mainly the clear coat) so as to obtain an IR spectrum truly representative of the composition of the coating. This may have a significant impact especially in instances where investigative leads need to be procured by library searching databases or via the use of chemometrics. A demonstration of this is shown in figures 5 and 6 . Figure 5 shows two IR spectra that have been extracted from a point in the outermost surface of the clear coat and a point near the base/clear coat interface of the paint cross-section obtained from the Toyota Camry. Each spectrum is dominated by the characteristic vibrational bands associated with acrylic binders as well as melamine and styrene. ${ }^{[6]}$ It is clearly evident that the main source of variation 
between the two spectra is the relative intensity of the peak attributable to melamine concentration, which could easily reduce the accuracy with which questioned paint samples could be identified against a library database search or a statistical model. For instance, the Paint Data Query (PDQ) software, which is maintained by the Royal Canadian Mounted Police, is a text-based database with a separate searchable IR spectral library. It was created by manually interpreting the IR spectra for the chemical components and proportions of each individual paint layer from a given sample. As the proportion of melamine has now been shown to fluctuate within the clear coat of a specified sample (as depicted in figure 5), the heterogeneity of the IR data obtained may invariably result in a discrepancy in the hits generated by the database.

$<$ Figure 5 $>$

Figure 5: IR spectra extracted from (a) an area of the clear coat previously shown to be affected by melamine migration and (b) a region of the clear coat unaffected by melamine migration. The highlighted peak corresponds to melamine and illustrates the variability in the proportion of melamine within the clear coat.

Furthermore, as mentioned previously, we have recently employed ATR FTIR in conjunction with chemometrics to characterise and classify a statistically large number of automotive clear coats obtained from a range of domestic and international manufacturers. ${ }^{[15]}$ As ATR spectra arise from only the first few microns $(0-3 \mu \mathrm{m})$ of the clear coat it is highly unlikely that these spectra will be affected by melamine migration issues. The same may not necessarily be true however for spectra obtained in transmission mode from paint cross sections. Our ATR-based statistical model revealed a number of small groupings which were linked to the source of the vehicle, thereby allowing information to be obtained regarding the possible manufacturer of the vehicle. If this model were to be applied to transmission spectra 
from paint cross sections however, an inconsistent melamine concentration across the different layers would most likely result in a misclassification of the sample, as demonstrated in figure 6 . The outcomes of this would be significant as this would result in misleading information being conveyed to law enforcement regarding the potential make and model of the offending vehicle.

\section{$<$ Figure 6 $>$}

Figure 6: 3-dimensional PCA scores plot for an ATR FTIR spectral dataset obtained from a large population of automotive clear coats. ${ }^{[15]}$ Spectra separate into classes representing the manufacturer source of the vehicle, however PC1, is significantly positively correlated to melamine and accounts for $92 \%$ of the variance in the dataset. As this is responsible for describing the differences in the relative abundance of melamine in the clear coat, if FTIR transmission spectra of Japanese vehicles showing melamine migration in class 1 were acquired from melamine contaminated areas of the clear coat, then the samples will attain more positive scores on PC1 resulting in potential misclassification with samples from class 4 (which contains different Japanese manufacturers) or 5.

Based upon these results, stringent analytical protocols need to be developed in order to ensure that the migration of paint additives does not affect the analysis and characterisation of paint layers. In particular in instances where IR microspectroscopy is employed to sequentially analyse individual layers of a paint cross section, it is vital that a sampling aperture size be chosen to limit the area of analysis towards the surface of the clear coat. For the remaining underlying layers the spectra should be measured as far from the adjacent layers as possible (i.e. mid-centre of the coating). It will also be efficacious to use as small a sampling aperture size as is feasible and take measurements from a number of separated spots. Furthermore, precautions must be taken when analysing thin peels or shavings of 
individual layers to ensure the sections are taken from an area truly representative of the composition of the coating and devoid from any melamine contamination.

\section{Conclusions}

Synchrotron FTIR imaging was utilised to examine the interlayer dissemination of paint additives within automotive paint specimens. The melamine additive was shown to consistently diffuse, when it was not present in the original equipment manufacturer formulation, from the basecoat layer into the outermost clear coat layer. Pigments were also found to diffuse into the adjoining layers. From a forensic science viewpoint, the outcomes of these results are significant as the relative abundance of melamine and pigments in the clear coat will vary greatly depending upon the region of the layer analysed. As a result, appropriate analytical protocols must be established to negate the effects of melamine and pigment diffusion, so as to obtain a true representation of the composition of the coating for forensic identification purposes.

\section{Acknowledgements}

Part of this research was undertaken on the infrared microspectroscopy beamline at the Australian Synchrotron, Victoria, Australia. The authors thank Kelvin Parker and the staff of Prestige Sunroofs WA (Kardinya, Western Australia) for the automotive paint samples; Nathan Karslake and Aidon Slaney (Department of Chemistry, Curtin University) for assistance in collection of samples, Dr Paolo Raiteri (Department of Chemistry, Curtin University) for his help with data processing and the Australian Synchrotron staff for their expert help and support. Mark Maric is supported by an Australian Postgraduate Award.

\section{References}

1. S. Bell, Forensic Chemistry, Pearson Prentice Hall, New Jersey, 2006. 
2. A.R.W. Jackson, J.M. Jackson, Forensic Science, Pearson Prentice Hall, Essex, 2004.

3. J. Bentley, Composition, manufacture and use of paint, In: B. Caddy (Ed.), Forensic Examination of Glass and Paint: Analysis and Interpretation, Taylor \& Francis, London, 2001, pp. 123-141.

4. J.M. Challinor, Paint Examination, In: I. Freckelton, H. Selby (Eds.), Expert Evidence, Lawbook, New South Wales, 1993.

5. ASTM, E1610 - 02, Standard Guide for Forensic Paint Analysis and Comparison, 2008.

6. A. Beveridge, T. Fung, D. MacDougall, Use of infrared spectroscopy for the characterization of paint fragments, In: B. Caddy (Ed.), Forensic Examination of Glass and Paint: Analysis and Interpretation, Taylor \& Francis, London, 2001, pp. 221-232.

7. T.J. Allen, Paint sample presentation for Fourier transform infrared microscopy, Vib. Spectrosc., 3(1992) 217-237.

8. J.P. Beauchaine, J.W. Peterman, R.J. Rosenthal, Applications of FT-IR/microscopy in forensic analysis. Microchim. Acta, 1(1988) 133-138.

9. M. Szafarska, M. Wozniakiewicz, M. Pilch, J. Zieba-Palus, P. Koscielniak, Computer analysis of ATR-FTIR spectra of paint samples for forensic purposes, J. Mol. Struct., 924-926(2009) 504-513.

10. J.M. Wilkinson, J. Locke, D.K. Lang, The examination of paints as thin sections using visible microspectrophotometry and Fourier transform infrared microscopy. Forensic Sci. Int., 38(1988) 43-52.

11. E.M. Suzuki, Forensic science applications of diffuse reflectance infrared Fourier transform spectroscopy (DRIFTS): IV. Direct analysis of metallic paints-sampling considerations, J. Forensic Sci., 34(1989) 164-179.

12. D.J. McEwen, G.D. Cheever, Infrared microscopic analysis of multiple layers of automotive paints, J. Coat. Technol., 65(1993) 35-41.

13. S.G. Ryland, T.A. Jergovich, K.P. Kirkbride, Current trends in forensic paint examination. Forensic Sci. Rev., 18(2006) 97-117.

14. G. Edmondstone, J. Hellman, K. Legate, G.L. Vardy, E. Lindsay, An assessment of the evidential value of automotive paint comparisons, Can. Soc. Forensic Sci., 37(2004) 147-153.

15. M. Maric, W. van Bronswijk, S.W. Lewis, K. Pitts, Rapid characterisation and classification of automotive clear coats by attenuated total reflectance infrared spectroscopy, Anal. Methods, 2012, DOI: 10.1039/C2AY25419K.

16. K. Flynn, R. O'Leary, C. Lennard, C. Roux, B.J. Reedy, Forensic applications of infrared chemical imaging: Multi-layered paint chips, J. Forensic Sci., 50(2005) 832841.

17. L.M. Miller, R.J. Smith, Synchrotrons versus globars, point-detectors versus focal plane arrays: Selecting the best source and detector for specific infrared microspectroscopy and imaging applications, Vib. Spectrosc., 38(2005) 237-240.

18. H. Bohnke, L. Avar, E. Hess, Analytical studies of light stabilizers in two-coat automotive finishes, J. Coat. Technol., 63(1991) 53-60. 
19. J.L. Dupuie, W.H. Weber, D.J. Scholl, J.L. Gerlock, Clearcoat analysis in isolated and multi-layer paint systems by confocal Raman microscopy, Polym. Degrad. Stab., 57(1997) 339-348.

20. W. Chang, T. Chen, C. Yu, J. Kau, Comparison of embedding methods used in examining cross-sections of automotive paints with micro-Fourier transform infrared spectroscopy, Forensic Sci. J., 1(2002) 55-60.

21. D.C. Creagh, J. McKinlay, P. Dumas, The design of the infrared beamline at the Australian Synchrotron, Vib. Spectrosc., 41(2006) 213-220.

22. G. Massonnet, W. Stoecklein, Identification of organic pigments in coatings: Applications to red automotive topcoats. Part II: Infrared spectroscopy, Sci. Justice, 39(1999) 135-140.

23. The first coating technology in the world that simultaneously reduces VOC and $\mathrm{CO}_{2}$ emissions, In: Social \& Environmental Report, (2006) 29-30. 
Figure 1:

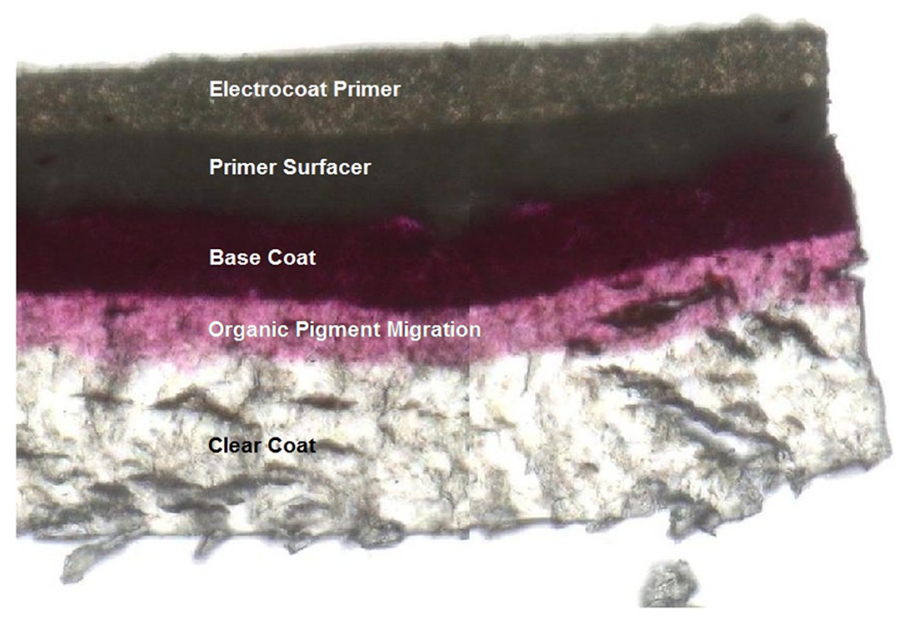

Figure 2:

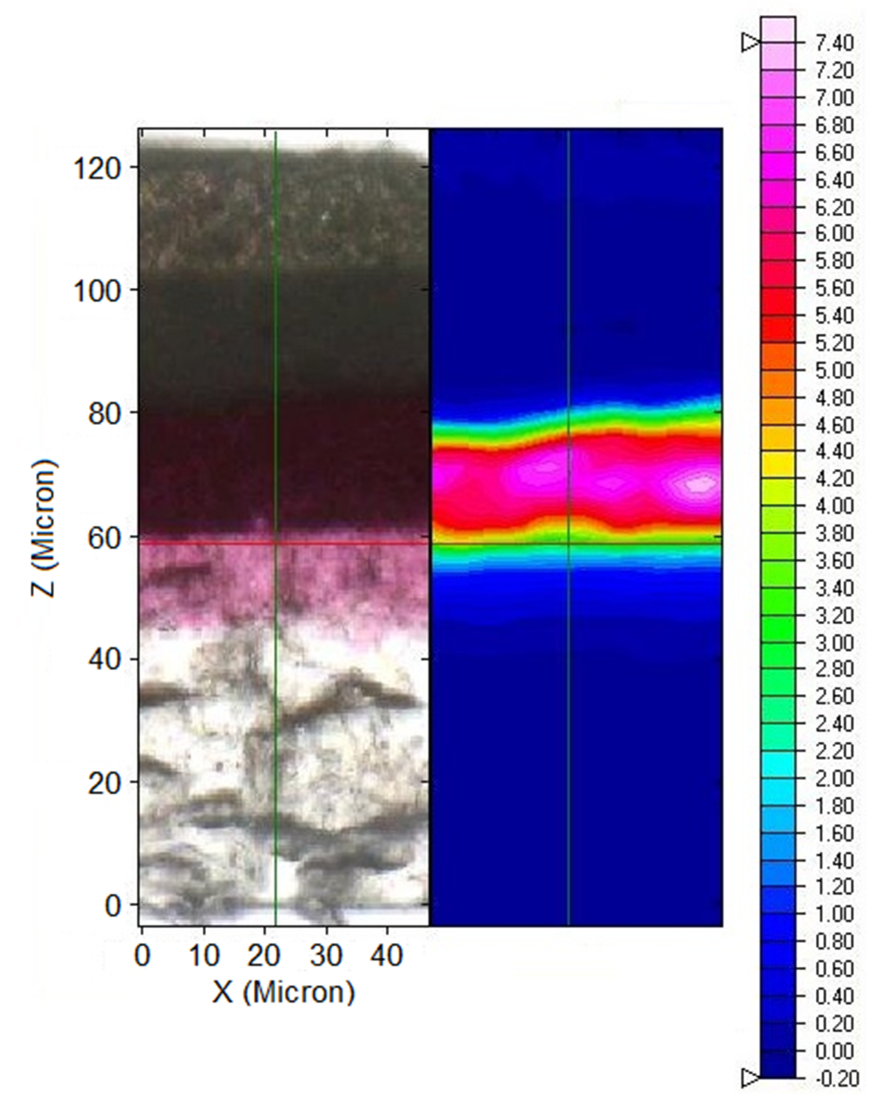


Figure 3:

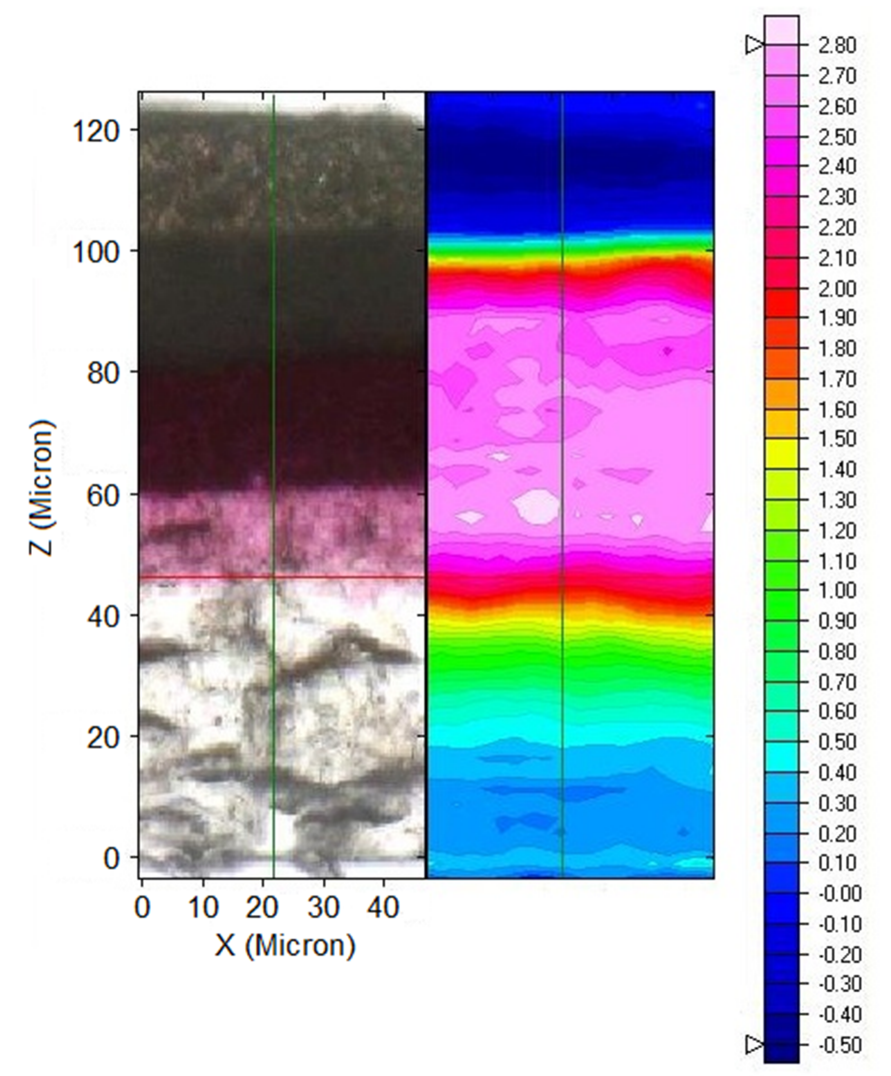

Figure 4:

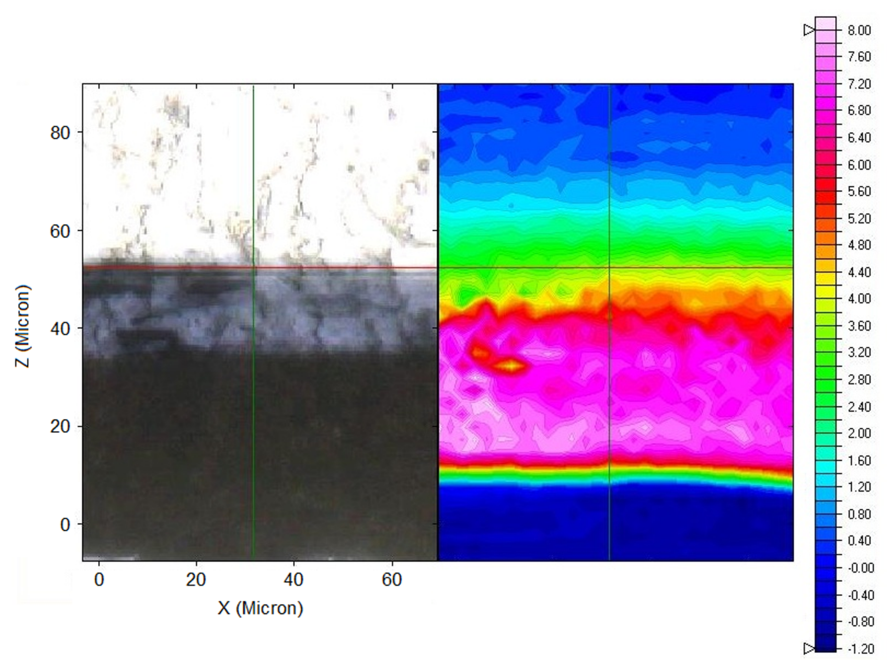


Figure 5:

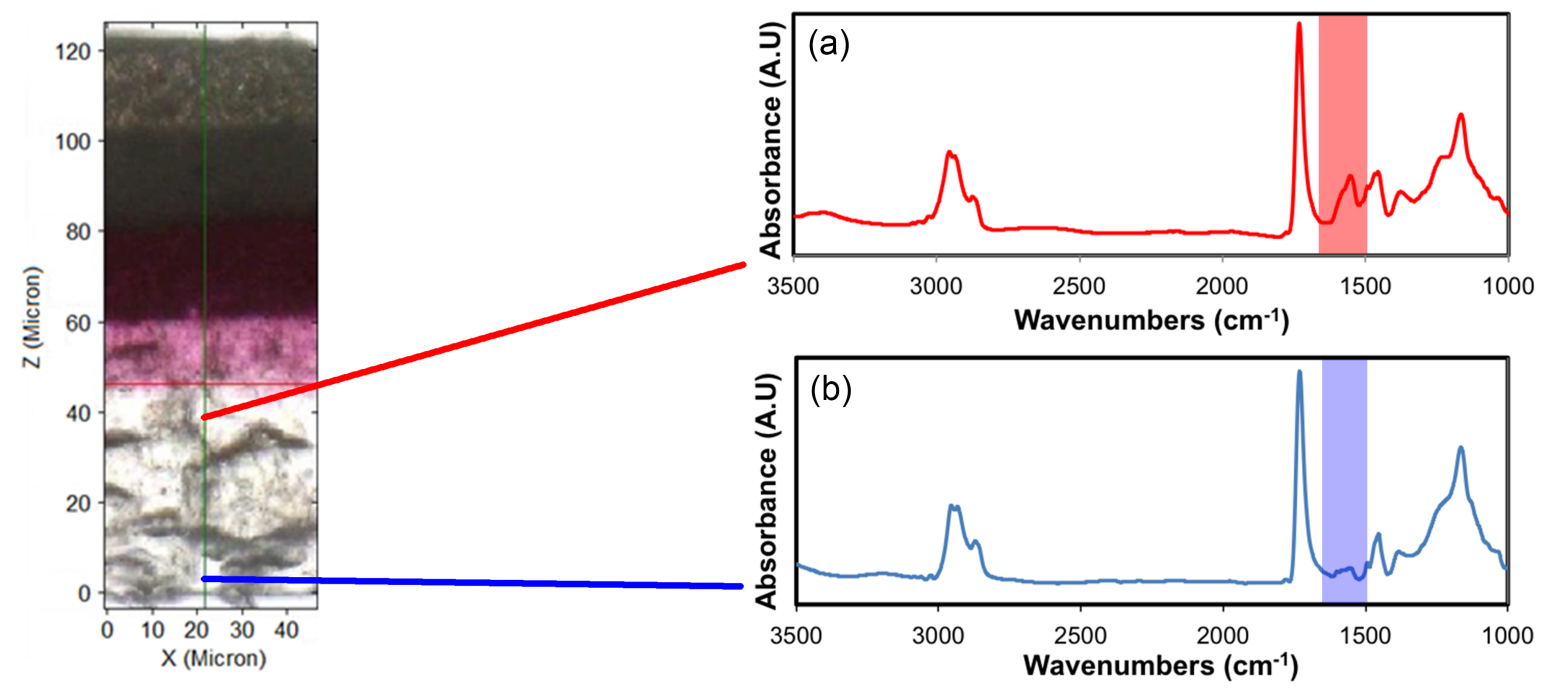

Figure 6:

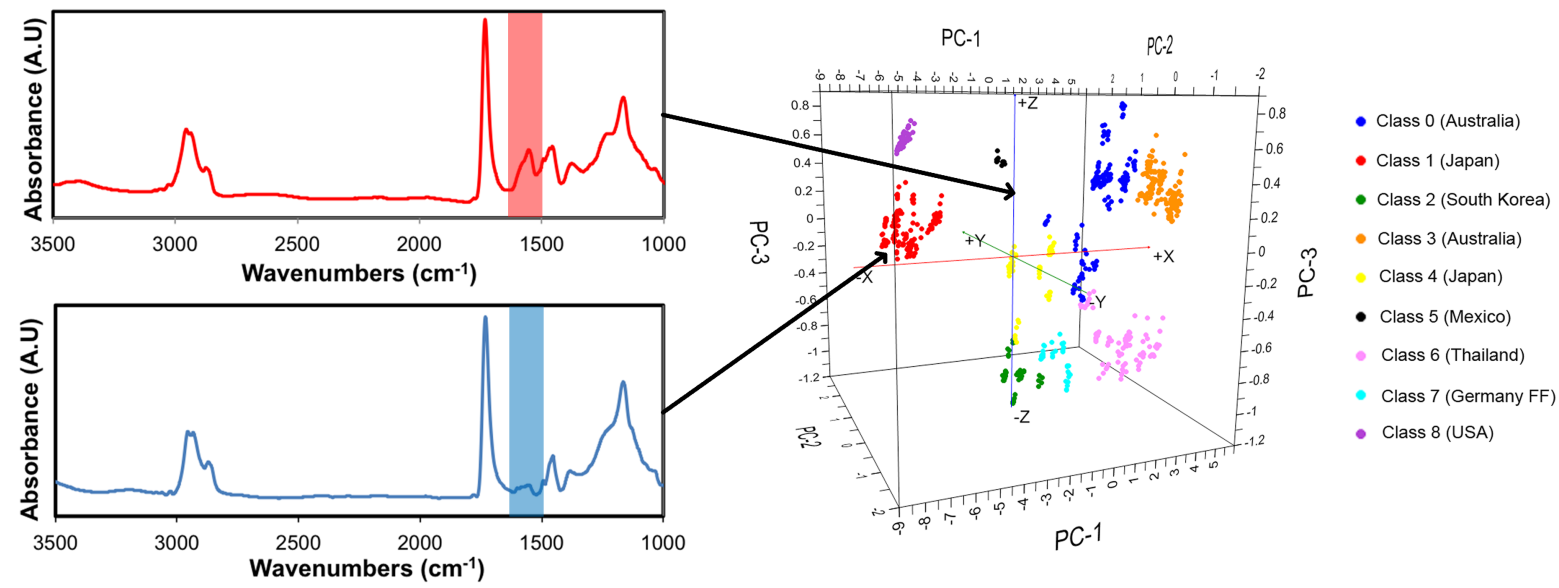

